\title{
Research on the Expression Form of Emotional Semantics in the Product Design
}

\author{
Jingjing Du \\ Suqian College \\ Suqian, China
}

\begin{abstract}
With combination of relevant knowledge of the product design, this article summarizes and analyzes the expression form of the emotion of the product. Through detailed analysis and summary of the product design case, it extracts four expression forms of the emotional semantics of product: shape, color, material, interactions. The design idea of "peopleoriented" is throughout the whole design process, makes the emotional semantic expression form of the emotion semantic become the medium of communication between people and product.
\end{abstract}

Keywords—emotional semantic; product design; shape; color; material; interaction

\section{INTRODUCTION}

In China, it has been conveying the people's worship to totems in that time from the "human face fish grain pottery basin" in the early Neolithic, spanning to handmade furniture of Ming and Qing dynasty periods which is exquisite carver and beautiful structure, especially the luxury decoration of the Qing dynasty furniture, they are not only the simple seating product, sleeping product, but more is the aesthetic taste of the people to the art in that time. To the modern industrial products, lamps and lanterns, tea set, kitchenware and tableware and etc., the designers use the each details of product to process and design, and inject the "human" factor to bring the new life to the products, make them able to "speak", make the consumers understand tacitly and feeling particularly more cordial, going into the external and connotation world of the products. The physical language and body language is the medium of human communication, but the shape, color, material, interaction is the medium of communication between the people and product that is the semantic form of the products.

\section{CONCEPT INTRODUCTION}

\section{A. Product Emotional Semantics}

The emotional semantic of the product: it is using "language" to express the emotional meaning of product to the consumers. In the late 20th century, the cultural value of the products was back to the front of our eyes. The products designed by the designers are no longer to be lifeless; bit contains a vivid era culture. The products have been expressing the emotion to us with their own unique "language", and the consumption structure of the people has also changed gradually from the material consumption to an informational consumption[1]. In the cultural context change with each passing day, the expression of the product has been further unlocked, and it is more laying emphasis on the emotions of the people. The designers are also trying to get the various ways, to make the products to respond to emotions of the consumers. The emotional semantic of the product is created in this situation.

\section{B. The Importance of Emotional Semantics of Product}

Product is the article specially designed for the people demand with purpose. It occupies the core status in the three major factors of the design system of "human - machine (product) - environment". The emergence of emotional semantic brings new opportunities for product designers, and provides the new options for the direction and entry point for the product design. The designer will give the product personalized "language" to disseminate information and to express emotions so that consumers can understand the product properties and the hidden meaning more clearly. In the book "the meaning of product morphology", three research goals are presented: The first purpose is to make the product "speak"; the second purpose is to make products more personalized; the third purpose is to make the product meaningful [1]. On the whole, the research purpose is to integrate the "peopleoriented" design idea into the product, to devote particular care to product design form of both "aesthetic perception" and "utility", and make the product can become the medium of communication.

\section{THE EXPRESSION FORM OFTHE EMOTIONAL SEMANTIC} IN THE PRODUCT DESIGN

\section{A. Shape Semantics}

Shape semantic is the most direct expression factor of the semantic meaning of the product, and the modelling is used as the expression carrier. Whether it is flat or solid, it is composed of simple point, line and face. When these elements are given a meaning in the design, they convey the product "language". In the product design, the shape semantic embodies two ways of manifestation mode- Explicit modelling semantic and implic it modeling semantic.

\section{1) Explicit modeling semantic}

The so-called explicit modeling semantic is namely through the simple observation to the product external, the user instantly can understand the function and operation mode of 
the product. In general, it is used as an auxiliary element to express the function and operation of the product through some pictures, symbols and symbols. Explicit modeling semantic is generally used more in public facilities and household appliances. The purpose is under no explanation of the specialized personnel, the product can also convey its own security, reliability and value and etc. make the customers easy to understand the meaning of the products.

"Fig. 1" and "Fig. 2" is a granger microwave oven that works up and down mode, different from the square side of the traditional microwave oven. When it comes to this product, the user will first be attracted to the design that it personalizes. Second, through observing the product, the user is easy to receive the explicit semantic meaning of the product. Safety: the metal case and reasonable structure color design give people steady and hard sense of safety; 2 . Versatility: when the user open the cover of the microwave oven, it can be found that it takes $45^{\circ}$ and $65^{\circ}$ and several swing-up door angle, and when $65^{\circ} \mathrm{swing}$-up open, it builds a large space, make it easy for the users to make food, also can be used as a similar fire cooking tool [2]; 3. Reliability: The product key is with text and icon elements and the automatic timer function, and brings the customers with reliable operation "instruction".

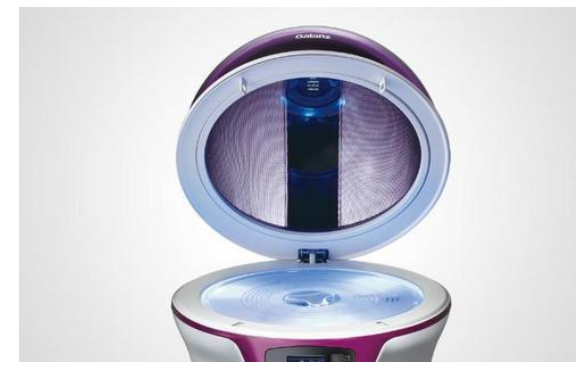

Fig. 1. Granger UOVO microwave (photo from net work).

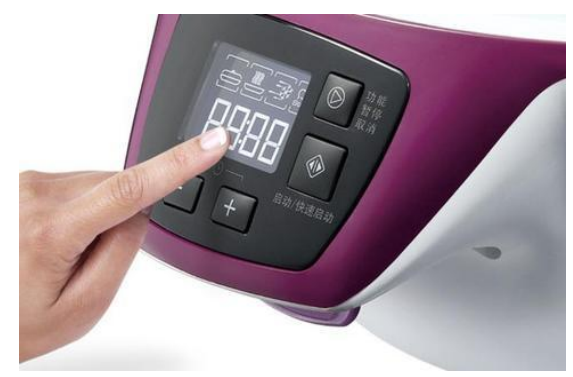

Fig. 2. Granger UOVO microwave (photo from net work).

"Fig. 3" and "Fig. 4" is the bionics design LED table lamp which is the design work of the author. It has the explicit modeling semantic which is embodied in: 1. Safety, animal form and resin material shell, bring people safety feeling of light and insulation. Multifunctional: the lamp is divided into up and down two lights namely the study lamp and the background lamp, both work and life without fail; 3 . Convenience: the desk lamp is with touch screen display, under WIFI environment, for the time, weather, and schedule of every day and the studying that is excessively using the eye, it will give reminder and etc., and provides the convenient life.

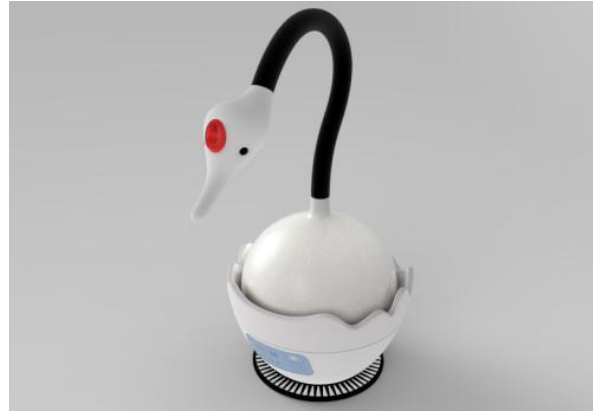

Fig. 3. LED lamp biomimetic design.

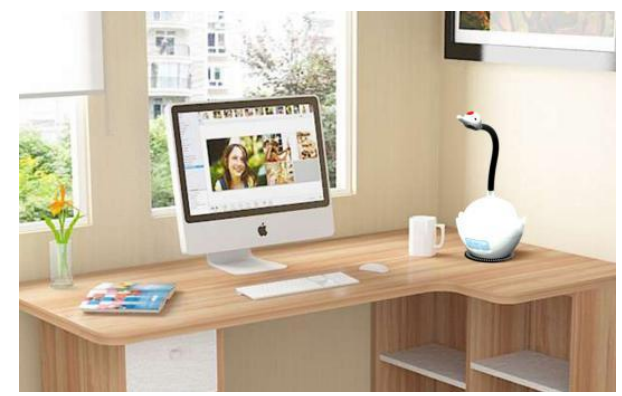

Fig. 4. LED lamp biomimetic design.

\section{2) Implicit modeling semantics}

Contrary to explicit modelling semantic, the Implicit modeling semantic is not simply to express product function and operation mode, it is more to want to through the interesting and personalized design product modeling, allows the user to make the depth association and imagination, so that to integrate into the inner spiritual world of the user, to convey a kind of emotional need.

In modern design, the large and small products have begun to apply the implicit modeling semantic. Applying the form bionics product in the product design is the most common convey mode of the implicit modelling semantic. It usually appears in daily necessities, food packaging, and the appearance of the home decoration class, to create unique visual enjoyment for the users. "Fig. 5" is "Honey, I'm Ho me!" key frame. The honeycomb is very chic and adorable. It can be placed on the wall of porch, matched with the key chain of hexagon. When entering the door, the owner of the hous ehold can hang the key on it. In Honey, I 'm Home! "Honey" means "honey" which has "dear" meaning, and "honey" means "home". The combination of both makes the implicit design semantic of this product - welcome home.

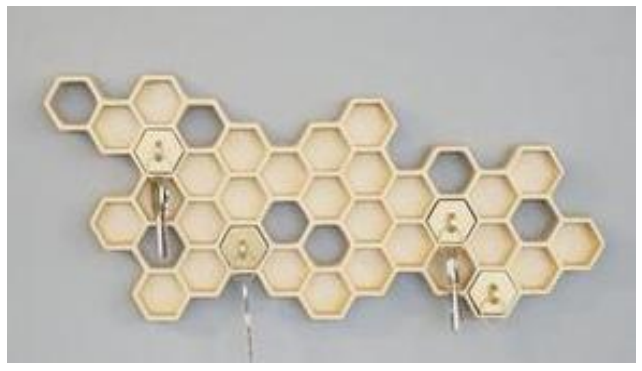

Fig. 5. Honey, I'm Home! Key chain (photo form net work). 
Take the author's design of "Fig. 3" as example, the modelling combines the red-crowned crane and the cracked eggshell, has a kind of graceful and clever feeling. When making conception of the whole lamp modeling, the pursuance makes the product more close to life. A side water and soil raises one party people, as the" from "He Xiang " children, the author more hopes that through this product, to provide the hometown people with the way of lovemaking on thing, affectionate by seeing thing, and also to show culture from $\mathrm{He}$ Xiang to other villages. So the Implicit modeling semantic is ----- Ho metown love.

\section{3) Color Semantics}

Color is one of the important elements of product design, mainly through changing the three elements of color, to give the product different "character" feature. So the color of radiating the charm has the vivid effect to the visual choice to the product. According to the statistics, the two major elements of modelling and color of stimulating the visual sense account for about $20 \%$ and $80 \%$ [3] respectively. It can be seen that the position of the color subjectivity is very high. In the product, the color semantic embodies three characteristics.

\section{4) Discrimination}

In modern design, the designers will differentiate the high degree color and apply it in the article, such as the sing of the toilet and bathroom in the plane. The facial cleanser and tea cup and other articles for use in the product, even if the product doesn't change in modeling, the consumers can distinguish the product from the color contrast for the people groups.

"Fig. 6" is the bathroom sign designed by the author. The design concept is using the element to distinguish the bathrooms of men and women. On color, the blue and red which has set in popular thinking are used for it. The consumers need not too attention to detail, can be the first time to make the corresponding choice. In the design, it is also added the four elements of leg hair, slippers and nail polish to enhance the distinguishing effect.
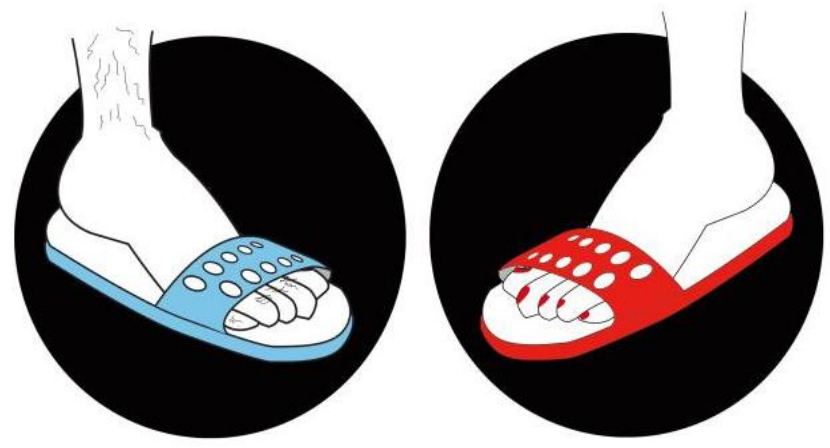

Fig. 6. Bathroom sign

\section{5) Warning mark}

On the basis of differentiation, color semantic also brings the function of warning. The different light has its specific wavelength of light, by using in light in the "traffic light", it is according to the order of the wavelength and red color is arranged first, with great capacity of penetrating into the medium, the strong capacity can be easy observed, so it is as the "stop sign"; Yellow color is relatively easier to differentiate then the orange, and the optical wavelength of yellow color is ranked at third in the seven colors, with large capacity of penetrating into the medium, the strong capacity can be easy observed, so it is as the "pass signal"

\section{6) Association}

In the development of color semantics, it not only has the visual impact of the surface, but also is going deeply into the emotional world of the consumers.

Mr. Wen Yiduo wrote in the poem of "color": "life is a piece of worthless white paper, since green color gave me development, red color gave me enthusiasm, yellow color gave taught me loyalty, blue color taught me nobleness, pink color conferred me hope, gray color presented me sorrow, black color has to add me to death". In the poem, from the single color, you can be as sociated with the inner emotional world. In the different periods of different regional culture, people have different psychological reactions. In Chinese traditional concept, yellow color represents authority -exclusive use for the ancient royal. Purple color represents the noble - exclusive use for the minister in ancient times. Red color represents joy -wedding banquet. White color represents solemn and stirring funeral... In the modern age, the associative sex of color semantics is richer, and it develops into two sides - positive and negative.

To sum up, the arising of color semantic is not by accident, but by the culture, society, science and technology, trend of the times.

For product designers, locating the color of the product should be established on the basis of its distinction, warning, and association, at the same time, analyze the different people groups, and finally integrate the color semantic in it perfectly.

\section{B. Material Semantics}

Material semantics are generally tactile and visual product under two kinds of sensory stimulation, any material has its own texture, luster, structure, texture, use on the product presents unique beauty. Without processing, the material has a soft, rough feel in touch, For example: textile materials, rubber materials, etc. Glass material, metallic material feels up to be very hard. The wood material, the ramie cotton material feels the rough; Silky materials and clay materials are smooth. In the absence of color, the material has a warm, light weight in sight. Marble and jade, for example, look cool. The clay materials and wood materials look relatively warm. Textiles and rubber look very light; and metal and jade materials look relatively heavy.

"Fig. 7" leisure chair: it is made of bamboo strip material, contracted and generous, especially suitable for outdoor or patio, so as to express close to the natural feelings. "Fig. 8" is called "Soft Light" creative lamps and lanterns, the main body material is made of polyurethane, and have highly flexible and security, users can be placed at random in any corner, can even use it as a pillow. 


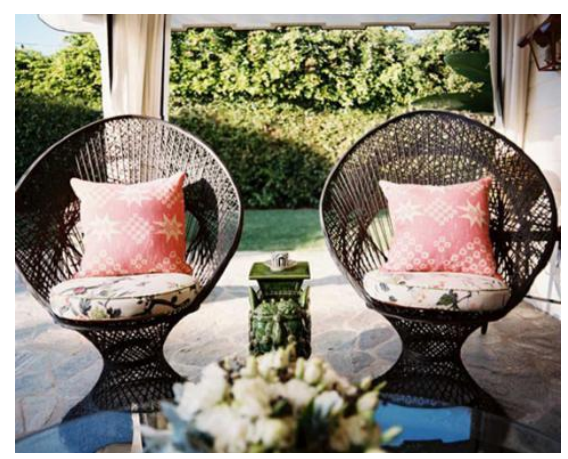

Fig. 7. Bamboo weaving chair (photo from net work).

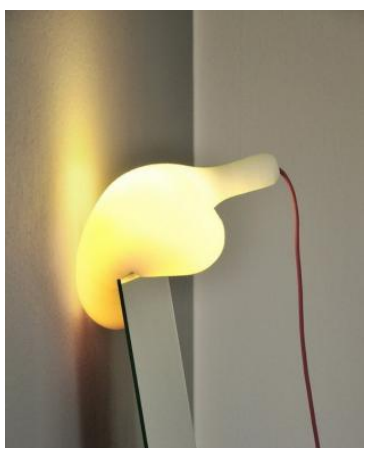

Fig. 8. Lamp "Soft Light"[4].

The material conveys its accompanied language form. When designing products, in order to satisfy the concept of users of recovering original simplicity, it needs to excavate the expressive force of the intrinsic material, and in order to enrich the emotion demand of the users, it needs to explore the new technology of materials processing and surface decoration [5].

\section{Interaction Semantics}

Interaction means communication and interaction, in a design conference in the 1980s of last century, the father of modern laptop -- (England), Bill · Moggridge firstly proposed the concept. The product is restricted to the emotional communication between people and products through the form of form, color and material, without the emotional interaction. With the continuous development of computer technology, some electronic products are integrated into our life, and the emotional interaction has been applied to this type of product such as: mobile phone, computer, household appliances, etc., the users can through the expression of product "language", not only can get the relevant information and services, but also more can obtain the real interactive fun. The "interactive semantics" arises at the historic moment.

\section{1) Gesture Interaction}

Gesture interaction is a very common way of interacting, and more directly expressing semantic. It's no longer the traditional meaning of through keyboard and mouse, but is realization through the touching screen and the smart pen. The use of hand gestures makes the life particularly relaxed easy, such as using your fingers to swipe your E-book to make the page turning, and drawing on a tablet with a smart pen.

\section{2) Speech recognition interaction}

Speech recognition interaction is the instruction expressed by the computer through voice to identify the user. Through the recognition of object, sound wave and voice, it is subdivided into voice recognition and speech recognition, such as voice control lamp, phonetic recognition of mobile phone, robot dialogue, etc.

In addition to this, under the driven by technology, there are interaction modes of eye recognition, fingerprint recognition. The diverse development of interaction means that the user has more interesting, emotional and diverse experiences. In the design, the user is the leader as well as the user of the interactive system. The interactive semantic requires more users to take the experienced as the design object, to provide the users with close interaction "language".

\section{CONCLUSION}

Through the analysis on the four forms of emotional semantic of the products, we can see that: in the future era, the demands of the consumers will be constantly increasing, and on the basis of the implementation of the product function meeting the demands of the consumers, you will focus on the emotional experience and satisfaction. It also makes the product design need the reasonably through four forms of emotional semantics to convey the emotion "language", make the "people-oriented" concept reasonably throughout the design process. When the emotion is well integrated into product, the product inevitably conveys the emotion.

\section{REFERENCES}

[1] Chen Caixia, Intercultural Awareness Cultivation in College English

[2] Chen Ju: The shape semantic of the product [M], Beijing institute of technology press, 2008. (9): 11-13

[3] Wang Luohao: The circular microwave oven UOVO Visual Studio of beyond [EB/OL].http:/kitchen.ea3w.com/25/258476.html, 2011.03.19

[4] Cài Ran: research on city logo color [J] Decoration, 2006. (6)

[5] Soft creative lamp design - Soft Light[DB/OL].http://www.jjxsp.com/archives/1285.html, 2014.09.22

[6] Zhou Hongjuan, Zhang Mingyue. The application of emotional semantic in the material of the product design [J/OL]. Design theory and art theory, 2007. (4) 